EPJ Web of Conferences 92,02087 (2015)

DOI: $10.1051 /$ epjconf/ 20159202087

(C) Owned by the authors, published by EDP Sciences, 2015

\title{
Experimental verification of computational model for wind turbine blade geometry design
}

\author{
Vít Štorch ${ }^{1, a}$, Jiří Nožička ${ }^{1}$, Martin Brada ${ }^{1}$, Jakub Suchý1 \\ ${ }^{1}$ Department of Fluid Dynamics and Thermodynamics, Faculty of Mech. Eng., CTU in Prague, Technická 4, Prague, Czech \\ Republic
}

\begin{abstract}
A 3D potential flow solver with unsteady force free wake model intended for optimization of blade shape for wind power generation is applied on a test case scenario formed by a wind turbine with vertical axis of rotation. The calculation is sensitive to correct modelling of wake and its interaction with blades. The validity of the flow solver is verified by comparing experimentally obtained performance data of model rotor with numerical results.
\end{abstract}

\section{Introduction}

A potential flow solver based on 3D panel method developed for rotating lifting surfaces by the author has been improved to account for unsteady flow features and parasitic drag. The ability of the solver to predict lifting forces generating thrust in an aircraft propeller had been verified already in previous research [1]. To check the performance of the solver in a much more involved scenario of a VAWT (vertical axis wind turbine), H-type rotor VAWT geometry has been proposed consisting of three wing-like blade elements. An experiment was carried out in order to verify numerical results.

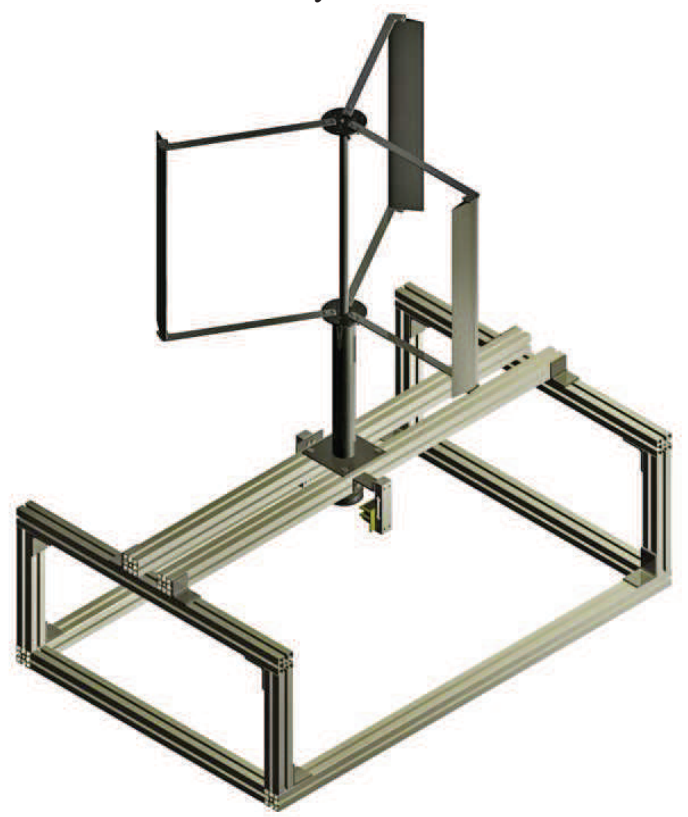

Figure 1. Visualisation of the VAWT model

\footnotetext{
${ }^{\mathrm{a}}$ Corresponding author: vit.storch@fs.cvut.cz
}

\section{Potential flow solver}

The potential flow solver is based on a 3D panel method described by Katz and Plotkin [2]. The velocity perturbation formulation of panel method is used. This results in triple the number of influence coefficients in comparison to more common potential formulation since the influence coefficients are in form of a vector as opposed to scalar coefficients in potential formulation of panel method. An advantage of this approach is fast computing of velocity fields that are readily available without the need of gradient operation on the scalar potential field.

The surface of each blade is discretized by a number of quadrilateral elements - panels. Both source and vortex ring singularities are placed on each body surface panel while only vortex ring panels are used for modelling the wake. In order to obtain a determined system of equations, the source strengths cannot act as unknowns and must be set beforehand. The goal of using source strength panels in addition to vortex rings is to remove most of the normal velocity required in the zero normal flow boundary condition. This reduces the amount of corrections by the vortex ring panels needed for fulfilling the boundary condition and eliminates some of the problems due to discretization and numerical errors. At the beginning of the calculation the source strengths $\sigma$ are determined by the condition (1) using the panel normal $\boldsymbol{n}$ and undisturbed local velocity $\boldsymbol{v}_{\text {loc }}$. This undisturbed local velocity consists of wind velocity at infinity and circumferential 'tip' velocity of the blade at actual position.

$$
\sigma=\boldsymbol{n} \cdot \boldsymbol{v}_{l o c} .
$$


The source influence coefficient matrixes are calculated for each pair of blades (including the influence of a blade on itself). For 3 blades with $N$ panels each, there are 9 matrixes, each containing $N \times N$ influence vectors. The proper evaluation of a flat source panel influence implemented according to Hess [3] is an order of magnitude more time consuming than that of a vortex ring. It is therefore beneficial to only rotate the previously calculated influence coefficient vectors after each time step, since the blades do not move with respect to each other, only rotate as a whole.

\subsection{Building the linear system of equations}

The calculation is initialized with only one wake row of panels at the trailing edge of each blade. The vortex ring influence coefficient matrixes are calculated including the wake with $N_{w}$ panels. For 3 blades, there are again 9 matrixes with $N \times\left(N+N_{w}\right)$ coefficients. Only the vortex strengths of surface panels are unknown, the vortex strengths of the $1^{\text {st }}$ row wake panels $\gamma_{1 W}$ are calculated from trailing edge panels $\gamma_{T E}$ upper, $\gamma_{T E}$ lower using Kutta condition [2] (2).

$$
\gamma_{1 W}=\gamma_{T E \text { upper }}-\gamma_{T E \text { lower }}
$$

The vortex strengths of the following wake panel rows (if they exist) are determined from the previous time step. This represents unsteady vortex shedding. Since the vortex strengths of all rows except the $1^{\text {st }}$ row are known, they are multiplied with their respective influence coefficients and placed on the right hand side of the system of equations. The system of equations for $M$ blades each with $N$ panels is built as follows:

$$
\begin{gathered}
\underline{A}=\left(\begin{array}{ccc}
C_{1,1}(N x N) & \ldots & C_{1, M}(N x N) \\
\vdots & C_{i, j}(N x N) & \vdots \\
C_{M, 1}(N x N) & \ldots & C_{M, M}(N x N)
\end{array}\right) \\
\bar{x}=\left[\bar{\gamma}_{1}(1 \ldots N), \ldots, \ldots, \bar{\gamma}_{M}(1 \ldots N)\right] \\
\bar{B}=\left[\bar{B}_{1}, \ldots, \bar{B}_{i}, \ldots, \bar{B}_{M}\right]^{\prime} \\
C_{i, j}(k, l)=\left[C x_{i, j}(k, l), C y_{i, j}(k, l), C z_{i, j}(k, l)\right] \cdot \boldsymbol{n}_{i, k} \\
\bar{B}_{i}=\left[\boldsymbol{n}_{i, 1} \cdot\left(\boldsymbol{v}_{i, 1 l o c}+\boldsymbol{v}_{i, 1 \sigma}+\boldsymbol{v}_{i, 1 \gamma, \text { wake }}\right), \ldots, \boldsymbol{n}_{i, N} \cdot(\ldots)\right] \\
\underline{A} \bar{x}+\bar{B}=0 .
\end{gathered}
$$

The coefficient matrixes $C_{i, j}(N \times N)$ entering the linear equation system are composed of scalars calculated by dot product of vortex ring coefficient vectors and the respective panel normal unit vector. The right hand side vector $\boldsymbol{B}$ is formed by the dot product of normal vector in each collocation point and the sum of local velocity with perturbation velocity due to sources on blades and due to vortex ring in the wake. The only exception is the first row of the wake. Its source strengths are unknown so its influence coefficients are added to proper members of matrix $\underline{A}$ according to Kutta condition (2).
Since each blade at one time step is subject to completely different local velocity field, there is no symmetry to take advantage of as in the case of propeller with axisymmetric flow.

After the linear system of equations is solved, it is possible to determine all vortex strengths and velocity distribution. For unsteady case, the pressure coefficient on the body surface [4] is prescribed as:

$$
c_{p}=1-\frac{\|v\|^{2}}{\left\|v_{\text {inf }}\right\|^{2}}+\frac{8 \pi}{\left\|v_{\text {inf }}\right\|^{2}} \frac{\gamma_{t}-\gamma_{t-1}}{\Delta t} .
$$

The resulting pressure is used for calculating the lift, induced drag and the total moment of force about the axis of rotation.

\subsection{Generating unsteady force-free wake}

After the surface vortex strengths have been calculated it is necessary to determine the velocity field in the wake. Again, vortex ring influence matrixes of size $N_{w} \times\left(N+N_{w}\right)$ are constructed. The influence of blade sources on wake panels is calculated using simplified source function, each panel representing a point source, in order to achieve faster execution time. This simplification is possible due to the fact that most wake panels are distant from the blade surface.

The goal of the force-free wake generation is to move the wake mesh in the direction of velocity field in the inertial system of coordinates (excluding the circumferential velocity). The local perturbation velocity can be reliably obtained only at collocation points in the middle of vortex rings. To get the velocity at panel corner points, velocity must be interpolated.

After the wake is moved in the direction of the velocity field, the first row of wake panels is stretched, since the wake stays attached to the trailing edge. The first row is then split into 2 rows, the second of which retains the calculated vortex strength and the first row still attached to the trailing edge receives empty data structure for vortex strength ready to be filled in the next time iteration by the Kutta condition.

\subsection{Discretization errors, other considerations}

A vortex ring panel is formed by four interconnected vortex filaments. The perturbation velocity influence on a nearby point approaches infinity as the distance of the point approaches zero. In the way panel method is constructed, trustworthy velocity vector can be obtained only at collocation point or at a reasonable distance (at least the length of a panel) from any singularity. This is not an issue when simulating simple flow such as that of a propeller or horizontal axis wind turbine, where the wakes do not intersect each other or the blades. Without any modifications, the result of intersecting wakes or surface geometries would be large or infinite jumps in vortex strength distributions and sudden breakup of the wake. To partly correct this behaviour, each vortex filament has a fixed velocity core, so the velocity does not approach infinity as the distance approaches zero. 


\section{Vertical axis wind turbine test case}

Vertical axis wind turbines (VAWTs) are often criticised for their lower efficiency and fatigue life issues. While pros and cons of such concept are an interesting topic itself, it is evident that such concept represents an excellent test scenario for evaluation of numerical model. The turbine starts its rotation with help of drag difference between blades facing the wind by the trailing edge and by the leading edge. As the tip speed ratio (TSR) $\lambda=\omega R / v_{\text {inf }}$ approaches 1 , the tangential component of the lift force starts to dominate and the turbine changes from poor drag powered device to efficient lift based device. The start-up is considered complete when $\lambda$ reaches 1 .

\subsection{Geometry}

The geometry of the turbine was constrained mainly by the available technology which allowed creating only straight blades. H-rotor concept was adopted. The $800 \mathrm{~mm}$ diameter rotor has three $500 \mathrm{~mm}$ long blades with $100 \mathrm{~mm}$ chord. The chord to diameter ratio $1 / 8$ is quite high which allowed higher Reynolds numbers and resulted in highly curved velocity field along the airfoil. The aspect ratio of each blade is 5 , which is too little for an efficient blade, but introduces noticeable 3D effects and tip losses. The airfoil was chosen NACA 0018 as a well-known and documented airfoil, which has been extensively tested at Sandia laboratory [5] for use with VAWTs. The blades are interchangeable for future testing of other airfoils.

\section{Numerical results}

\subsection{Single rotating blade}

The single rotating blade was modelled to verify correct wake modelling and evaluate the impact of the blade number on results. As can be seen in figure 2, the wake starts to roll up on each side with direction depending on the angle of attack. The moment calculated from pressure distribution on the blade surface throughout the whole revolution is smooth except when the blade intersects its own wake. There is a velocity jump when passing the wake sheet which results in inconsistencies in surface strength calculations, since the neighbouring panels may be each at different wake sides, which produces unexpected vortex strengths. These jumps are occurring only in one or two instances per whole revolution and may be smoothed out by a simple routine.

\subsection{Three rotating blades}

For the case of three rotating blades, limited number of panels was used. The execution time grows with the square of panel number. As a compromise surface discretized with 220 panels and maximum wake size 10x120 panels was used.

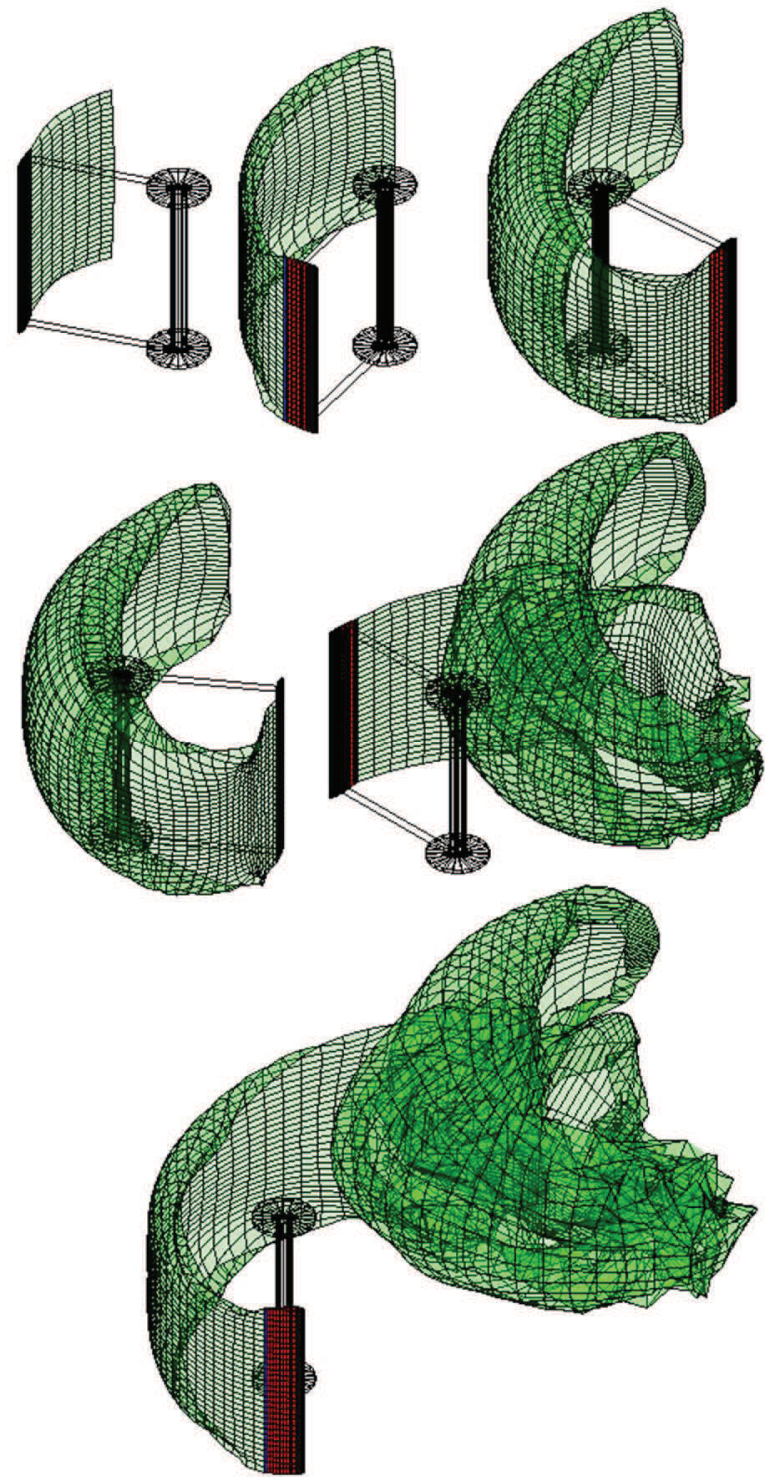

Figure 2. Evolution of the single blade wake. $\lambda=2$.

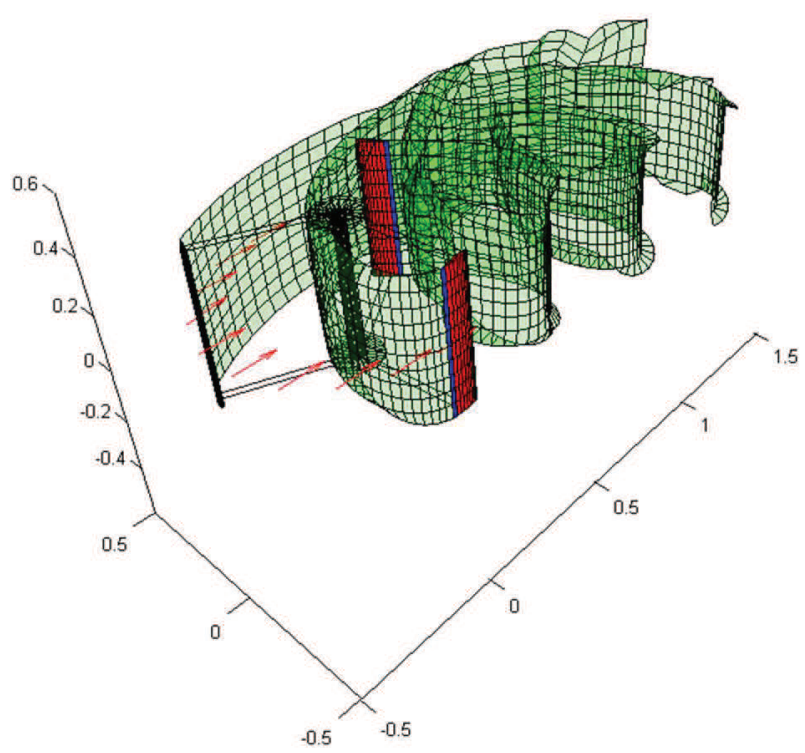

Figure 3. Evolution of three blade wakes. $\lambda=2$. 
The wake was initialized for each tip speed ratio with zero length and evolved for 3 revolutions. Only the last revolution was evaluated to obtain the average moment. Since the blade passes now at least three wakes during single rotation, jumps in velocities and forces were more prominent. This was more significant with increasing $\lambda$. It is also apparent that the blade generates much smaller moment during the downwind half of rotation. This is in part due to the lower available velocity, but also due to symmetric airfoil, which does not act as symmetric in strongly curved velocity field.

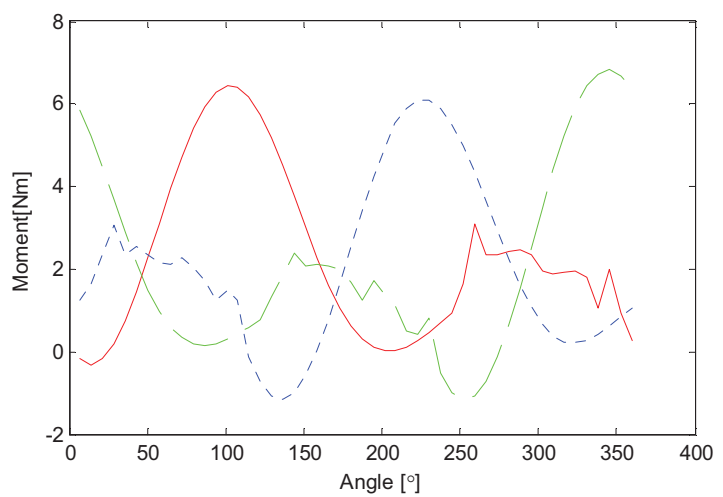

Figure 4. Moment from each blade during one revolution, $\lambda=2$.

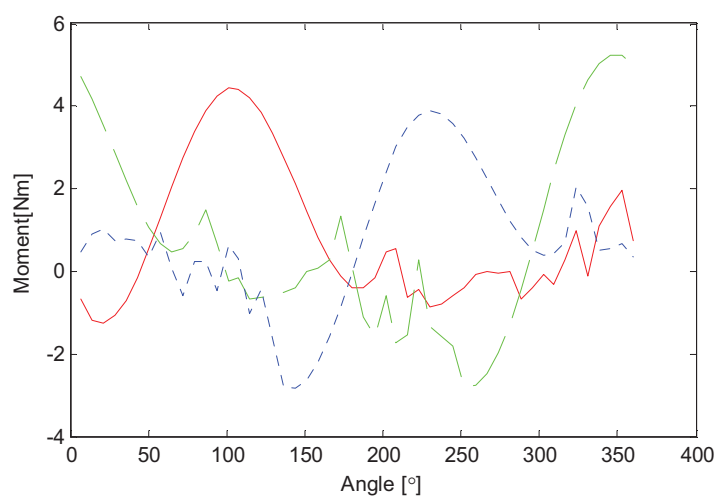

Figure 5. Moment from each blade during one revolution, $\lambda=4$.

\section{Experimental setup}

\subsection{Blade strength and airfoil accuracy}

Although the model is small scale and no significant power was expected, initial calculations have revealed tremendous forces acting on the blades. This is due to the fact that higher Reynolds numbers were required to reach a region where the airfoil loses most of its misbehaviour resulting from laminar bubbles and transition effects in low Reynolds regime. Maximum design parameters were $v_{\text {inf }}=18 \mathrm{~m} / \mathrm{s}$ and $\lambda=3.5$, which results in centrifugal acceleration of $10^{3} \mathrm{~g}$. Therefore extremely light and strong blades were required. The blades are made of vacuum formed epoxy-glass skin on a foam core. The core was cut using hot wire technique on a CNC machine. One of the manufactured blades was tested for maximum bending moment and after failure the undamaged parts were cut to test airfoil accuracy.
For accuracy test, the cut blade section was photographed with contrasting background and edited to 2 bit $\mathrm{B} \& \mathrm{~W}$ bitmap. The data was then loaded using MATLAB script and compared against original NACA coordinates.

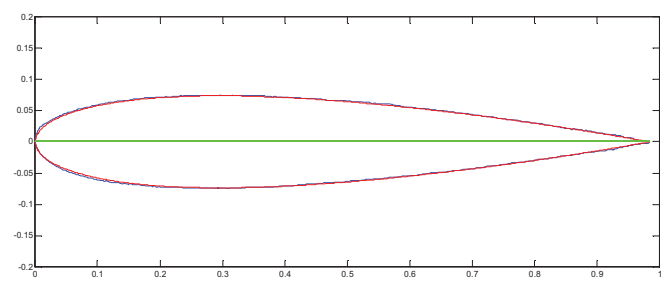

Figure 6. Comparing coordinates (red), and actual airfoil (blue).

\subsection{Measurement platform}

The turbine is placed on a rigid platform made of aluminium profiles designed to fit into the wind tunnel test chamber without interfering with the flow. Due to wind tunnel not being available as expected, the measurements were performed, for the time being, on an automobile roof. The measured quantities were time, frictional moment, angular speed and wind velocity. All the measured quantities were recorded in $5 \mathrm{~Hz}$ interval on a laptop.

The moment is measured by a pair of force sensors connected by a cord wrapped around a pulley on the rotor shaft. The tension of the cord is servo controlled by the operator.

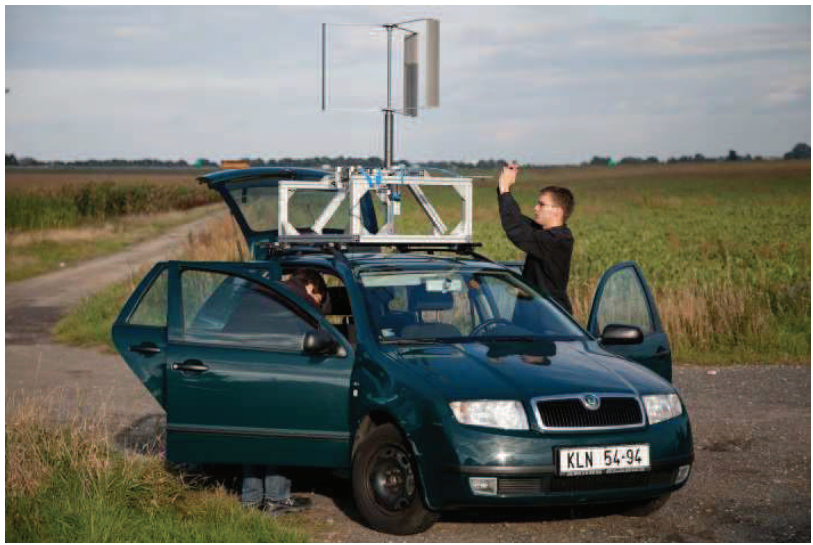

Figure 7. Experimental setup during field tests.

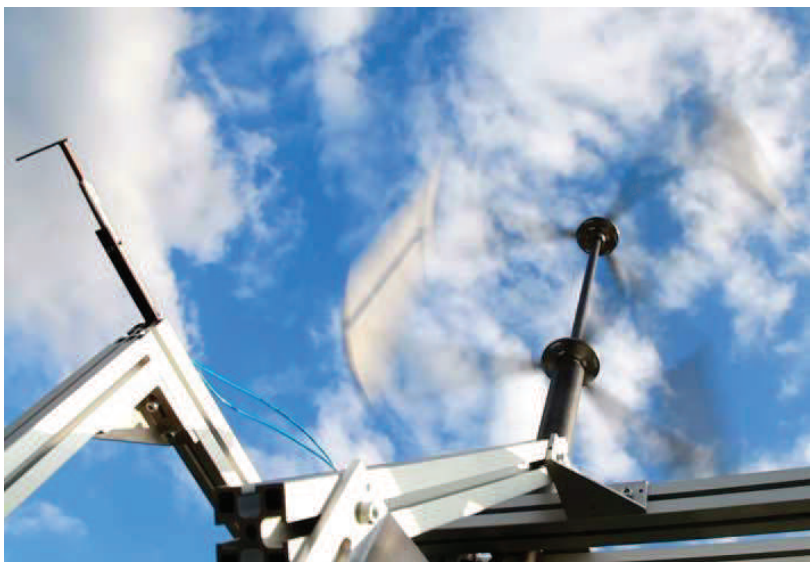

Figure 8. Running turbine. Note Pitot-static probe in the corner. 
It was crucial that the data were obtained during calm weather. This was met during the last of four measurement days. The wind velocity was below $0.5 \mathrm{~m} / \mathrm{s}$.

Each run was conducted on a straight $2 \mathrm{~km}$ long strip with smooth pavement. The driver was maintaining constant speed, while the operator increased slowly the load on turbine, until the turbine stopped, or the maximum achievable moment was reached. This was repeated for different velocities in the range $10 \div 45 \mathrm{~km} / \mathrm{h}$.

\subsection{Corrections for bearing and arm drag loses}

To be able to compare data against numerical results, bearing friction and blade arm drag had to be considered. The arms are made of $4 \mathrm{~mm}$ thick aluminium and have rectangular profiles. During rotation, each arm travels in the direct wake of the preceding arms. It was necessary to measure both bearing friction and aerodynamic drag experimentally. Under the assumption that the bearing friction moment $M_{b}$ is constant and the moment due to aerodynamic drag can be calculated as $M_{a}=C d_{a} \omega^{2}$, where $C d_{a}\left[\mathrm{~N} \mathrm{~m} \mathrm{~s}^{2}\right]$ is drag coefficient of turbine valid only for presented turbine under specified conditions, such as standard air density. It is possible to determine these constants by simply measuring the deceleration characteristics of an unloaded turbine without blades. The model for deceleration is simple:

$$
\frac{d \omega}{d t}=\frac{-M_{b}-C d_{a} \omega^{2}}{J_{(\text {no blades })}} .
$$

The moment of inertia of the rotating parts of the turbine without blades was calculated using Autodesk Inventor $J_{\text {(no blades })}=0.0350 \mathrm{~kg} \mathrm{~m}^{2}$. The moment of inertia with blades is $J_{(V A W T)}=0.07148 \mathrm{~kg} \mathrm{~m}^{2}$.

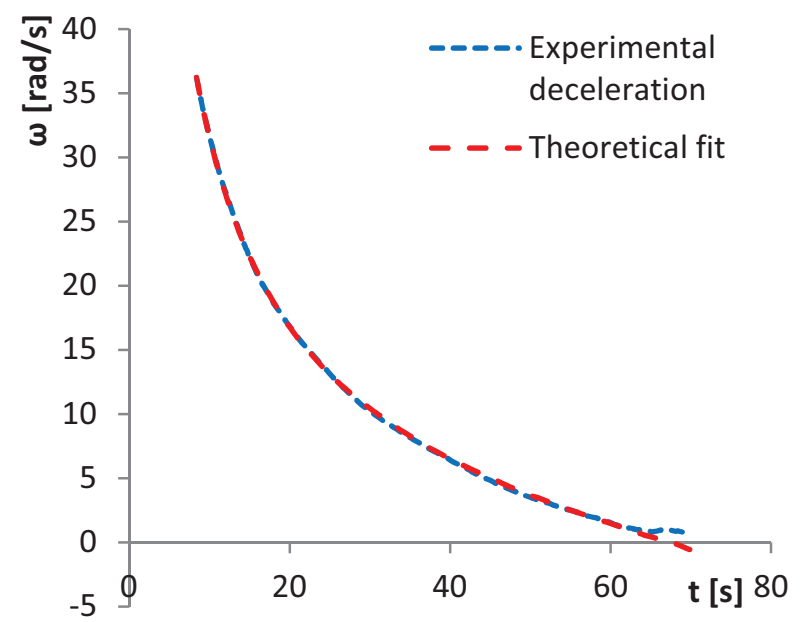

Figure 9. The experimental deceleration and theoretical fit.

From the deceleration, the constants were determined by least square fit as follows: $C d_{a}=7.8 \cdot 10^{-5} \mathrm{~N} \mathrm{~m} \mathrm{~s}^{2}$, $M_{b}=3.1 \cdot 10^{-3} N \mathrm{~m}$. It must be noted that the physical model of constant friction and constant drag coefficient can be fitted to the actual data very well.

\subsection{Data processing}

All the data were filtered by a simple moving average covering 5 samples to remove highest frequencies. Due to slightly fluctuating breaking moment, velocity and angular speed, the resulting output moment had to be corrected for energy being conserved or released due to change in angular momentum. The total moment generated by the blades including all corrections at time $t$ is calculated as:

$$
M(t)=M_{m}+M_{b}+C d_{a} \omega^{2}+J_{(V A W T)}\left(\frac{\omega_{t}-\omega_{t-1}}{\Delta t}\right) .
$$

Where $M_{m}$ is the measured moment. This model assumes that the wind velocity does not have any effect on the arm drag.

\section{Results of the experiment}

\subsection{Self start}

The model turbine was able to self-start in the wind magnitude above $5 \mathrm{~m} / \mathrm{s}$. For measuring performance in lower wind velocities, the turbine was self-started (term for starting without external power and reaching $\lambda=1$ ) at higher wind velocity and then the airspeed was reduced. Sample self-starting sequence without external load at wind speed $8.5 \mathrm{~m} / \mathrm{s}$ is in figure 10 . Note the almost instant acceleration to working tip speed ratio after reaching TSR $\lambda=1$.

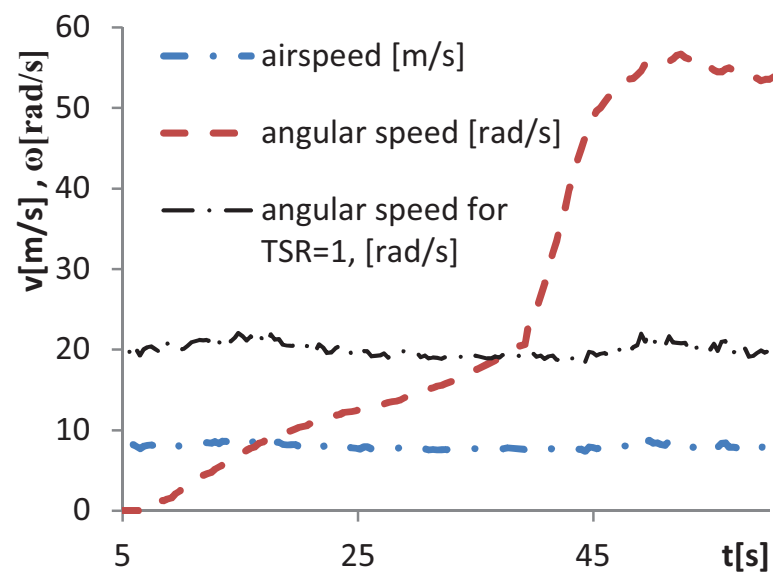

Figure 10. Self-starting sequence.

\subsection{Power curve}

The results were transformed into dimensionless coefficients $\lambda$ and $c p$. The power coefficient $c p$ is the ratio of generated mechanical power $M \omega$ to the total kinetic energy flux through the area $A$ swept by the blades.

$$
c p=\frac{M \omega}{\frac{1}{2} \rho A v^{3}} .
$$




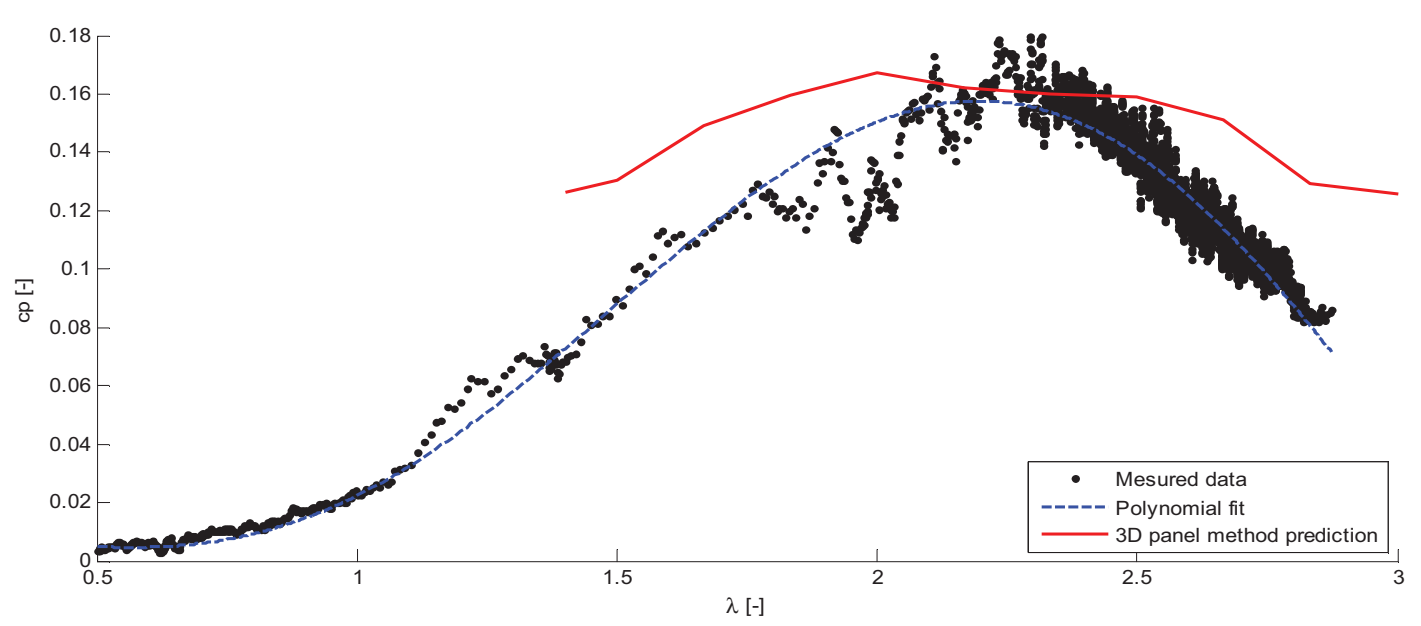

Figure 11. Power curve of the model VAWT. Cp vs. $\lambda$.

The dimensionless quantities are plotted in figure 11, together with the results of numerical calculations.

\section{Conclusion}

The proposed panel method is able to predict the peak power coefficient. The power curve resulting from the calculation has flatter shape suggesting that some of the aspects of the flow were not simulated properly by the flow solver. The high blade solidity needed for higher Reynolds numbers of the experiment resulted in low peak $\lambda$, which in turn means higher angle of attack. Panel method tends to over predict the lift in such scenarios. For higher $\lambda$, a longer wake might be necessary in order to account for the local wind velocity reduction due to power extraction. With increasing $\lambda$, the blade-wake interaction becomes a problem as seen in figures (4-5). The jumps in velocity and resulting forces have to be removed by a more sophisticated algorithm. Finer mesh is beneficial for the results of calculation. However, some code optimization would be required. The solver is currently running in MATLAB with parallel influence coefficient calculations using $\mathrm{C}++$ converted functions.

Some important aspects of the VAWT turbine design were brought to attention during this study. The simulation has revealed that the higher chord to diameter ratio of the turbine seriously affects the performance of the symmetrical airfoil and that a cambered airfoil would be more efficient. The amount of camber should account both for local velocity field curvature and downwind vs. upwind local wind velocity difference. The effect of the rotating arms that hold the blades cannot be neglected as it consumes almost half of the output power of the model.

For future work, cambered airfoils should be considered, with lower solidity to obtain higher tip speed ratios. The arms should be fitted with fairings to reduce drag.

\section{Acknowledgement}

This work was supported by the Grant Agency of the Czech Technical University in Prague, grant No. SGS 14/057/OHK2/1T/12

\section{References}

1. J. Filipský, V. Štorch, Comparison of propeller analysis methods and experimental data, Eng. Mech 2014, pp.172-175 (2014).

2. J. Katz, A. Plotkin, Low speed aerodynamics (2001)

3. J. L. Hess, Final Technical Report MDC J5679-01, Calculation of Potential Flow About Arbitrary Three-Dimensional Lifting Bodies (1972)

4. D. Ashby. Potential flow theory and operation guide for the panel code PMARC_14. (1999).

5. R. E. Sheldahl, P.C.. Klimas. Aerodynamic Characteristics of Seven Symmetrical Airfoil Sections Through 180-Degree Angle of Attack for Use in Aerodynamic Analysis of Vertical Axis Wind Turbines. (1981). 